\title{
Valor de la Marca en la Aceptación Sensorial de Consumidores en Cuatro Tipos de Pan Tajado Blanco
}

\author{
Claudia D. De Paula, Carlos A. García y Alba M. Durango \\ Universidad de Córdoba, Programa de Ingeniería de Alimentos, Facultad de Ingeniería, Carrera 6 No. 76- \\ 103, Montería - Córdoba, Colombia (e-mail: cdepaula@correo.unicordoba.edu.co; \\ adurango@correo.unicordoba.edu.co; cagarcia@correo.unicordoba.edu.co)
}

Recibido Feb. 22, 2013; Aceptado Abr. 17, 2013; Versión final recibida Ago. 30, 2013

\begin{abstract}
Resumen
Se estudió el efecto de cuatro marcas de pan tajado blanco sobre la calidad percibida por los consumidores a través de respuestas hedónicas. Se aplicó un test ciego y otro test con información de marca a 50 consumidores utilizando una escala de 1 a 7: "me disgusta extremadamente" (valor 1) y "me gusta extremadamente" (valor 7). Los datos se analizaron con análisis de varianza (ANOVA) y Test de Duncan. La variabilidad individual de los consumidores fue evaluada aplicando un mapa de preferencia interno explicando la variación de $70.4 \%$ para el test ciego y $63.5 \%$ para el test con información. Los consumidores tuvieron correlación positiva con el primer componente principal, atribuyendo notas más elevadas para la marca $X$, indicando el efecto de marca. El análisis de clasificación ascendente jerárquica, generó tres clúster en el test ciego y cuatro en el test con información. El género y edad de los consumidores tuvieron un efecto sobre las respuestas hedónicas.
\end{abstract}

\section{Value of Brand on the Consumer Sensory Acceptance of Four Types of White Sliced Bread}

\begin{abstract}
The effect of four white-sliced bread brands on the quality perceived by consumers through hedonic response was studied. A blind test and a test including brand information were carried out with 50 consumers using a scale from 1 to 7 : "I extremely dislike it" (value 1) and "I extremely like it" (value 7). Data were analyzed using analysis of variance (ANOVA) and Duncan Test. The Individual variability of consumers was evaluated using internal preference map that explaining the variation of $70.4 \%$ for the blind test and $63.5 \%$ for the test with information. Consumers had a positive correlation with the first principal component, indicating higher grades for the $\mathrm{X}$ brand, indicating the effect of brand. Analysis of hierarchicalascending classification generated three clusters in the blind test and four in the test with information. Gender and age of consumers had an effect on the hedonic responses.
\end{abstract}




\section{INTRODUCCIÓN}

La percepción del consumidor es una interacción de las características inherentes al producto y la condición personal socio-económica, sicológica, fisiológica y cognitiva. Cuando el consumidor está en el mercado la decisión de compra es influenciada por la marca y ésta ejerce un efecto en la respuesta de la evaluación sensorial. Las recientes investigaciones han prestado atención al efecto de la marca, el precio, aspecto socio-culturales o la preferencia general en la evaluación sensorial del alimento y el uso de métodos estadísticos para estudiar la influencia en el comportamiento del consumidor (Minim et al., 2012) y muestran como la segmentación de los consumidores juega un papel significante en la elección de un alimento (Grace et al., 2010). Considerando la aproximación multidimensional del valor de marca realizada por Aaker (1994), que identifica cinco activos de la marca con capacidad para crear valor (reconocimiento del nombre y símbolos de la marca, percepción de calidad, otras asociaciones de la marca, fidelidad a la marca y, finalmente, otros activos de la marca), se tratará de verificar la correspondencia entre las valoraciones hedónicas asignadas por el mercado a las diferentes marcas sin conocimiento del nombre y con conocimiento. Una hipótesis de cómo la información puede influir en las notas afectivas sobre un producto es a través de su influencia en las expectativas del consumidor (Hui, 2006). Así, la aceptación se incrementa en los casos donde la expectativa es alta y decrece en los casos donde la expectativa es baja.

Un área importante para el desarrollo estratégico de un producto y la identificación de posibles segmentos de consumidores es el uso de los Mapas de preferencia (MDPREF) que es un procedimiento estadístico que considera las individualidades del consumidor y su identificación con las preferencias de las muestras evaluadas (Viana et al., 2012; Minim, 2010). Los mapas de preferencia interno permiten complementar el estudio sensorial afectivo de un producto explicando las preferencias del consumidor y determinar las más aceptadas por la mayoría de estos (De Souza et al., 2010). El objetivo de esta investigación fue verificar el valor de cuatro marcas de pan tajado blanco en la aceptación sensorial de los consumidores.

\section{MATERIALES Y MÉTODOS}

Fueron evaluadas cuatro muestras comerciales de pan tajado blanco con menos de un día de hacerse fabricado, simbolizadas por Marca X, Marca Y, Marca Z y Marca W. La Marca X es tradicional y reconocida en el mercado y la MARCA $Z$ es nueva en el mercado. Se vincularon 50 consumidores habituales del producto. Las pruebas fueron realizadas en cabinas individuales del Laboratorio de Análisis Sensorial de Carulla Vivero S.A con sede en Bogotá, cumpliendo con todos los requisitos de la NTC 3884. Las tajadas de pan fueron servidas en platos plásticos blancos desechables, presentados en forma monadica y aleatoria. Se realizaron dos sesiones, un test ciego y un test con información de la marca, en donde se aplicó una prueba de aceptación para evaluar la impresión global de las muestras, empleando una escala hedónica de 7 puntos variando entre los términos hedónicos "Me gusta extremadamente" (7), "Me gusta mucho" (6), "Me gusta" (5), "Ni me gusta ni me disgusta" (4), "Me disgusta" (3), "Me disgusta mucho" (2) y "Me disgusta extremadamente" (1).

Los datos fueron analizados usando el software XLSTAT Versión 2009.3.02, empleando análisis de varianza y el test de Duncan para encontrar diferencias significativas entre las medias de los datos del test ciego e informado para cada muestra de pan $(\mathrm{p} \leq 0,05)$. Un análisis de componentes principales (ACP) fue aplicado a las respuestas hedónicas para observar la variabilidad individual de los consumidores y la Clasificación Ascendente Jerárquica (CAJ) para obtener clúster de consumidores con características homogéneas de preferencia. Las similitudes fueron calculadas sobre la base de la distancia Euclidiana y a los clúster de consumidores fue aplicado el ANOVA y el test de comparación de medias de Fisher-LSD $(p \leq 0,05)$. El análisis descriptivo fue evaluado en cada clúster para establecer las características demográficas con relación al género y al rango de edades de 21 a 30, 31 a 40 y 41 a 50 años.

\section{RESULTADOS Y DISCUSIÓN}

El análisis de varianza mostró diferencias significativas entre las notas de satisfacción cuando fueron valoradas en el test ciego $(p<0,0001)$. Estas valoraciones sensoriales no fueron tan grandes como los obtenidos en el test con información. La Figura 1a muestra que el rango de medias fue de 4,34 a 5,20 en el test ciego y solo dos grupos diferentes de panes fueron establecidos $(p \leq 0,05)$.

Este comportamiento sugiere que, cuando el consumidor no fue consciente de la identidad del pan, no emitió su concepto influenciado por la marca y posiciono su preferencia en la parte media de la escala hedónica. Esto probablemente debido a las características similares de las muestras de pan tajado blanco. 

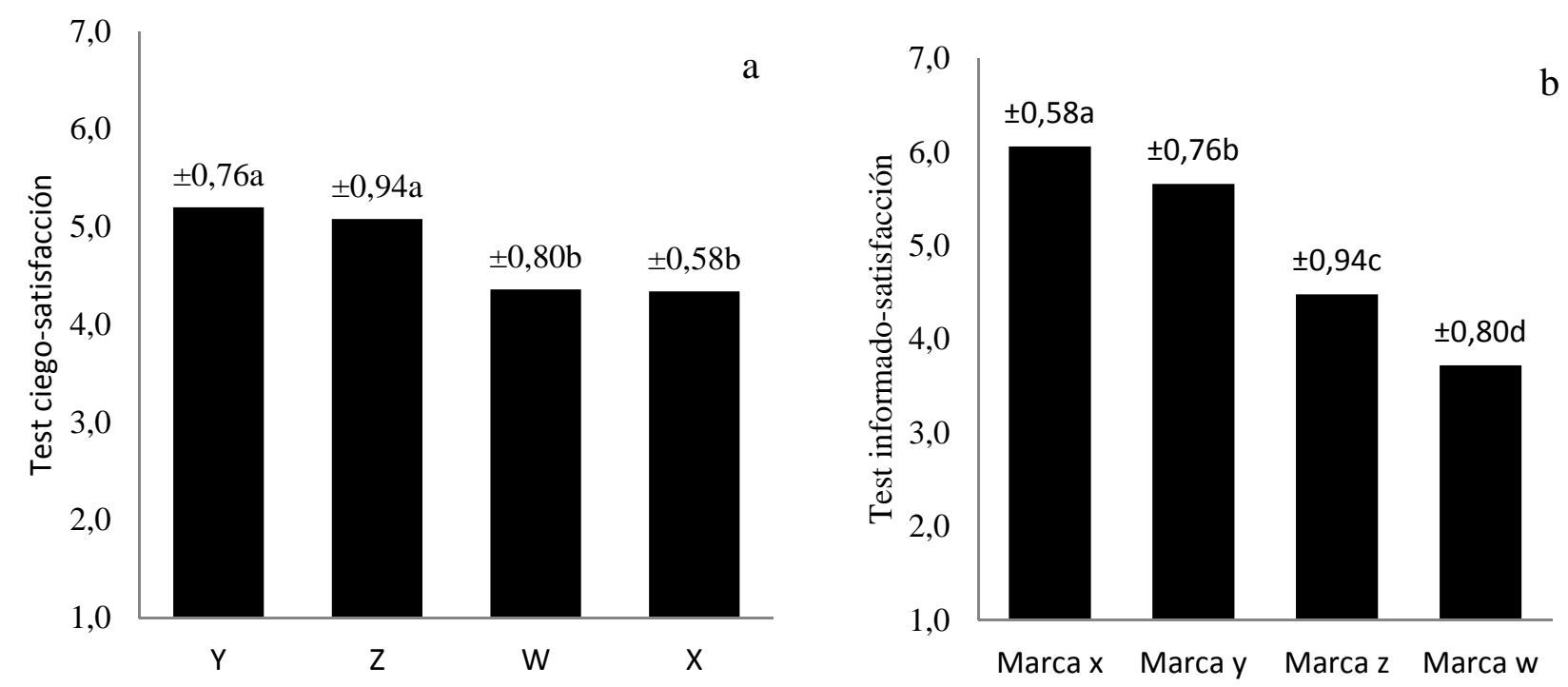

Fig. 1: Notas medias del grado de satisfacción de test ciego (a) y con información (b) de las 4 marcas de pan tajado. Medias con la misma letra no tienen diferencia significativa (Duncan, $p \leq 0,05$ ).

El test con información de la marca presento diferencias significativas $(p<0,0001)$ entre las marcas de pan tajado. Las diferencias de los grados de satisfacción entre las marcas es un poco más pronunciado que en las obtenidas en el test ciego. El rango de medias fue de 3,72 a 6,06 en las notas sensoriales, presentando cuatro grupos significativamente diferentes (Duncan, $p \leq 0,05$ ) en marcas de pan (Figura 1b).

La marca $X$ fue la más preferida en el test con información y la de menor preferencia en el test ciego junto con la marca $W$. El conocimiento de la marca incrementó la nota en las marcas $X$ y $Y$ y la redujo en las $Z$ y W. Cuando el nombre de la marca es conocida genera una distorsión en la opinión del consumidor. Es interesante observar que $\mathrm{W}$ se mueve a la nota más baja correspondiente al rango de "ni me gusta ni me disgusta" a "me disgusta". Monaco et al. (2004) mostraron que el conocimiento de la marca no afecta la percepción de los atributos sensoriales, sugiriendo que las expectativas generadas por el nombre de la marca son esencialmente en la escala hedónica usada; sin embargo, Cotes y Muñoz (2010) indicaron que los atributos intrínsecos y extrínsecos más valorados por los que prefieren marcas de distribuidor en jamón ibérico en comparación con los que prefieren marca de fabricante, son similares salvo en la preferencia por "marcas reconocidas", que está más presente en los que prefieren marca de fabricante. Sigurdsson et al. (2009) reportaron que la mezcla de mercado está diseñada para estimular e influenciar la demanda de los consumidores manipulando las variables asociadas con las marcas. Esto dice que el pan tajado con la marca comercial W, reduciría la intensión del consumidor por el producto.

Al observar el efecto del conocimiento de la marca (Tabla 1) las diferencias significativas cuando se restan las medias de los t-test, se evidencia que la única que no tuvo efecto fue la marca $Y$ y la que presentó un efecto positivo fue la marca X. El test ciego (Figura 2a) es explicado por los dos componentes principales en un $70,43 \%$ de la variación existente entre las marcas de pan en relación al gusto general. Se forman dos grupos, siendo el primero compuesto por la muestra $\mathrm{Y}$ y $\mathrm{Z}$, y el segundo por $\mathrm{X}$ y W. Los consumidores están localizados próximos a la región de las muestras que más les gusto, dándole notas altas. Son pocos los consumidores no asociados con los cuadrantes de los grupos.

Tabla 1: Diferencia de las medias del test Informado (I) menos el Ciego(C) ${ }^{* *} \mathrm{p}<0,001$ (t-test)

\begin{tabular}{|c|c|}
\hline Marca & I-C \\
\hline W & $-0,64^{* *}$ \\
\hline$X$ & $1,72^{* *}$ \\
\hline$Y$ & 0,46 \\
\hline$Z$ & $-0,60^{* *}$ \\
\hline
\end{tabular}


EI MDPREF efectuado cuando se dieron a conocer las marcas, los dos componentes principales explicaron un $63,48 \%$ (Figura $2 \mathrm{~b}$ ) de la variación en el gusto de las marcas de pan. Hay una distribución más homogénea de los consumidores en los cuatro cuadrantes y un reposicionamiento de las marcas $\mathrm{Y}, \mathrm{Z}$ y W, excepto la Marca $X$ que se mantuvo en su cuadrante. Los consumidores localizados en la región central de la figura no están correlacionados con los dos componentes, consecuentemente contribuyen poco a discriminar las muestras de pan en relación al conocimiento de la marca (MacKay, 2005).
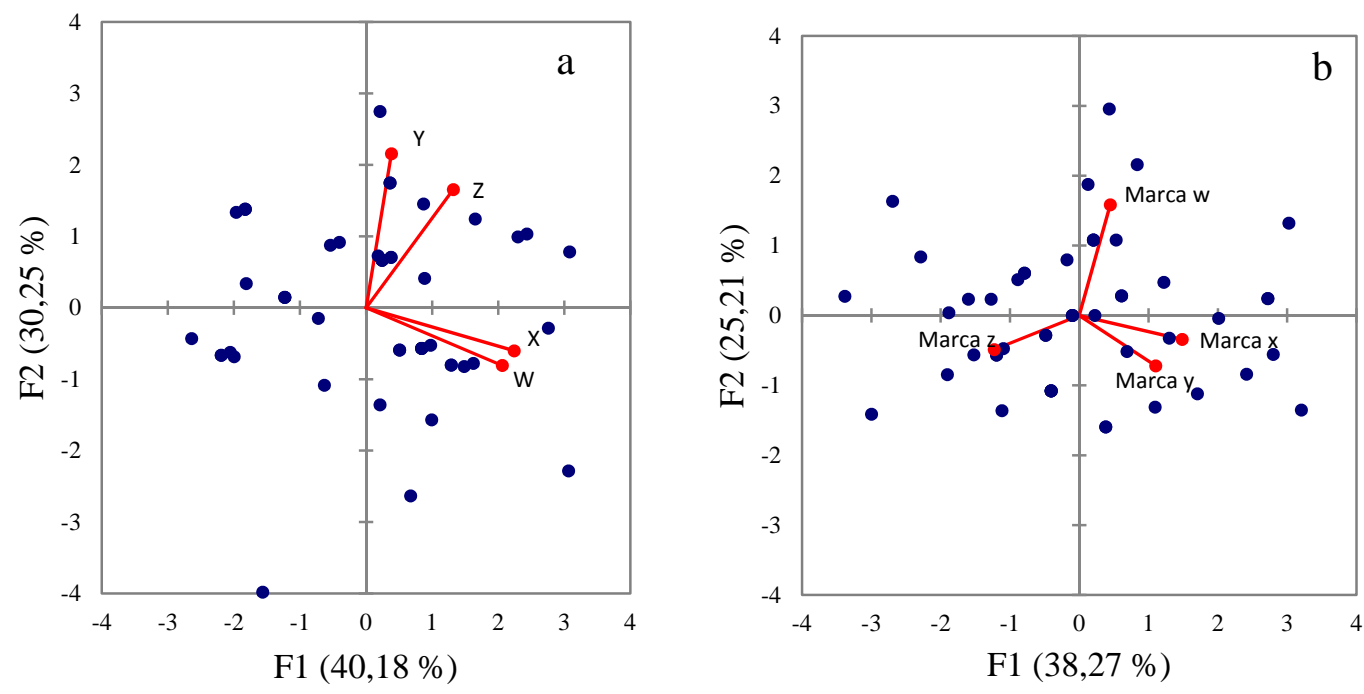

Fig. 2: Mapa de preferencia interno del test Ciego (a) y test con Información (b).

La Clasificación Ascendente Jerárquica (CAJ) de los datos sensoriales identifico los consumidores que fueron homogéneos en su preferencia (Tabla 2) para el test ciego con 3 clústeres y el test informado con 4 clústeres. La segmentación de los consumidores, muestra que al jerarquizarlos cuando se conoce la marca del pan tajado, la que mayor efecto tuvo en la respuesta fue la Marca $X$ que en promedio obtuvo un 6,18 correspondiente a "me gusta mucho" respecto a los 3 clústeres en X con una nota de 4 , asociados a "ni me gusta ni me disgusta".

Tabla 2: Media de preferencias por Clasificación Ascendente Jerárquica de consumidores.

Medias con la misma letra no tienen diferencia significativa (LSD, $p \leq 0,05$ ).

\begin{tabular}{cccccc|cccccc}
\hline \multicolumn{7}{c|}{ CAJ-Ciego } & \multicolumn{7}{c}{ CAJ-Informado } \\
\hline Clúster & No. & X & Y & W & Z & Clúster & No. & M-X & M-Y & M-W & M-Z \\
\hline 1 & 8 & $2,75 a$ & $5,62 \mathrm{a}$ & $3,25 \mathrm{a}$ & $4,37 \mathrm{a}$ & 1 & 12 & $6,00 \mathrm{a}$ & $4,75 \mathrm{a}$ & $3,16 \mathrm{a}$ & $4,58 \mathrm{a}$ \\
2 & 19 & $4,52 \mathrm{~b}$ & $5,84 \mathrm{a}$ & $4,68 \mathrm{~b}$ & $5,57 \mathrm{~b}$ & 2 & 20 & $5,70 \mathrm{~b}$ & $5,60 \mathrm{~b}$ & $4,30 \mathrm{~b}$ & $4,60 \mathrm{a}$ \\
3 & 23 & $4,73 \mathrm{~b}$ & $4,52 \mathrm{~b}$ & $4,47 \mathrm{~b}$ & $4,91 \mathrm{a}$ & 3 & 9 & $6,00 \mathrm{a}$ & $6,44 \mathrm{c}$ & $3,11 \mathrm{a}$ & $5,00 \mathrm{a}$ \\
& & & & & & 4 & 9 & $7,00 \mathrm{c}$ & $6,22 \mathrm{c}$ & $3,77 \mathrm{c}$ & $3,55 \mathrm{~b}$ \\
Media & 4,00 & 5,33 & 4,13 & 4,95 & & & 6,18 & 5,75 & 3,59 & 4,43 \\
\hline
\end{tabular}

Las respuestas hedónicas de los 3 segmentos de consumidores en el test ciego no son superiores a la indiferencia en la preferencia (ni me gusta ni me disgusta) de las cuatro marcas de pan; en el caso del test informado, los 4 segmentos expresan apreciaciones favorables sobre todo en las marcas $\mathrm{X}$ y $\mathrm{Y}$, demostrando que hay una influencia de las marcas comerciales en la intención de compra de los consumidores, identificando las variables que incrementan la discriminación y mejora la efectividad de las marcas para reforzar el interés del consumidor por comprar una marca específica (Foxall et al., 2007; Semeijn et al., 2004). Las notas medias de preferencia de cada clúster para las 4 marcas de pan tajado en los dos test fueron usados para elaborar un MDPREF (Figura 3) donde los dos componentes principales explican el $100 \%$ de la variación en la preferencia de las 3 clústeres en el test ciego y el $78,67 \%$ en el test con información. Esto permitió la representación visual de la preferencia de los segmentos de consumidores (Drake et al., 2009; Pages, 2005; Nestrud y Lawless, 2008).

La separación espacial de las muestras de pan sugiere la existencia de 3 grupos acorde a la preferencia de los consumidores en el test ciego (Figura 3a), siendo un grupo formado por las marcas $\mathrm{W}$ y $\mathrm{X}$, otro por la marca $\mathrm{Z}$ y otro por la marca $\mathrm{Y}$. Los clústeres asociados por lo menos con un componente principal consideran diferencias en la preferencia de las muestras. Para los segmentos 2 y 3 hubo correlación 
positiva con el primer componente, indicando respuestas asociadas a una mayor preferencia a las marcas $X$ y Z; los segmentos 1 y 2 se relacionaron más a la marca $Y$. En el test informado observase los segmentos formados por la marca W, la $Z$ y la agrupación de $X$ y $Y$. El clúster 2 no se encuentra asociado en el cuadrante de alguna marca, aportando este grupo de consumidores respuestas homogéneas con respecto a las 4 marcas (Figura $3 b$ ); es decir, no hay una influencia de la marca en la opinión de estos consumidores.
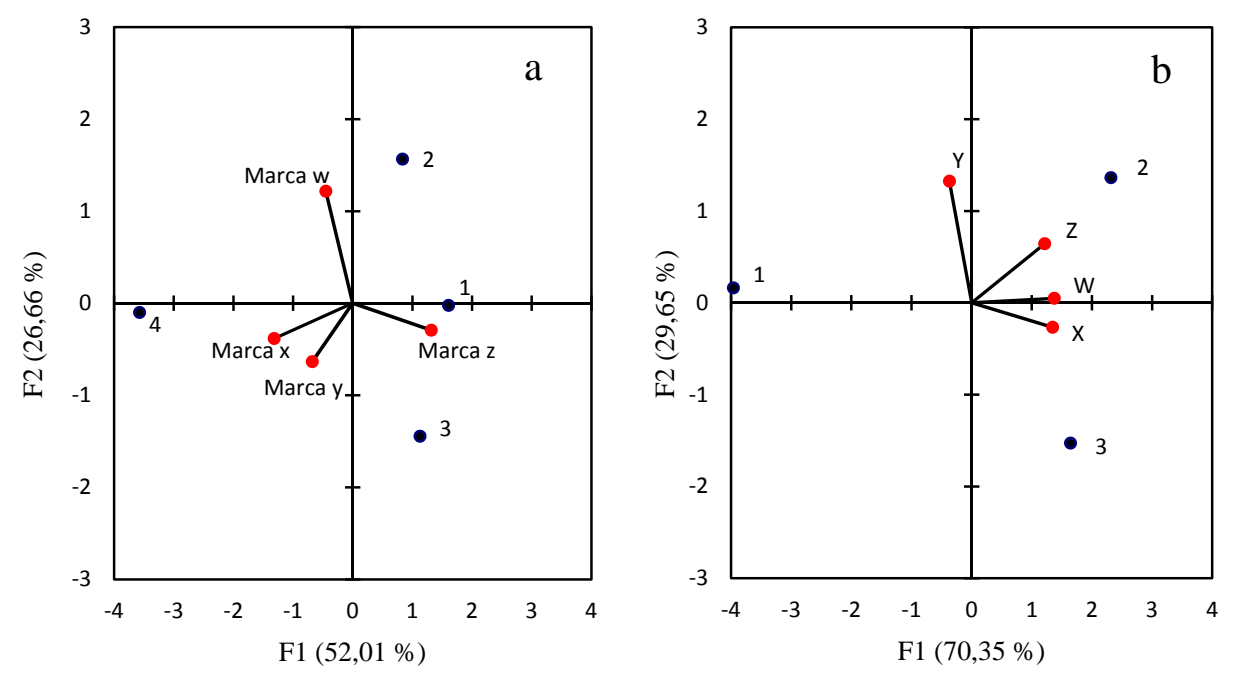

Fig. 3: Mapa de preferencia interno para los clúster de consumidores obtenidos por CAJ del test ciego (a) y test con información (b).

Las características demográficas de los consumidores tales como género y edad influyeron en las respuestas hedónicas de los consumidores respecto a los test (Tabla 3). La edad en particular fue un factor determinante en la preferencia de las muestras de pan tajado. Consumidores jóvenes del clúster 1 en el test ciego y en el informado, expresan su preferencia por el pan X cuando reconocen la marca; en contraste con el segmento 2 que está formado por las 3 edades agrupadas de consumidores que dieron respuestas más homogéneas.

Tabla 3: Perfil demográfico de las clases de consumidores Jerarquizados.

\begin{tabular}{cccc|cccc}
\hline \multicolumn{4}{c|}{ Clúster - Ciego } & \multicolumn{4}{c}{ Clúster - Informado } \\
\hline Genero & 1 & \multicolumn{1}{c|}{2} & \multicolumn{1}{c|}{3} & 1 & \multicolumn{1}{c}{2} & 3 & 4 \\
\hline Femenino & $75,0 \%$ & $63,1 \%$ & $52,1 \%$ & $75,0 \%$ & $45,0 \%$ & $55,5 \%$ & $77,7 \%$ \\
Masculino & $25,0 \%$ & $36,8 \%$ & $47,8 \%$ & $25,0 \%$ & $55,0 \%$ & $44,4 \%$ & $22,2 \%$ \\
\hline Edad & & & & & & & \\
\hline 21 a 30 & $62,5 \%$ & $78,9 \%$ & $78,2 \%$ & $91,6 \%$ & $70,0 \%$ & $88,8 \%$ & $55,5 \%$ \\
31 a 40 & 0 & $5,2 \%$ & $8,7 \%$ & 0 & $5,0 \%$ & $11,1 \%$ & $11,1 \%$ \\
41 a 50 & $37,5 \%$ & $15,7 \%$ & $13,0 \%$ & $8,3 \%$ & $25,0 \%$ & 0 & $33,3 \%$ \\
\hline
\end{tabular}

Los segmentos formados mayoritariamente por consumidores femeninos expresan respuestas hedónicas diferentes de un test a otro, observándose que este tipo de consumidores es influenciado por la marca del pan tajado. Los consumidores femeninos jóvenes son influenciados por el conocimiento de la marca en sus respuestas hedónicas sobre el pan tajado blanco. Reicks (2006) reporto que las diferencias por género y edad en las notas de consumidores femeninos fueron más altas $(p<0.05)$ que las dadas por los hombres y que generalmente son incrementadas por el incremento de la edad, cuando evaluó los factores que afectaban la decisión de compra de filete de cerdo asado y en bistec. La publicidad funciona si deja o fortalece trazos y asociaciones en la memoria para la marca en la mente de los consumidores, de tal manera que la marca venga a la mente cuando surja una oportunidad de compra (López et al., 2010). Este mismo comportamiento fue observado en el presente estudio.

\section{CONCLUSIONES}

Las notas hedónicas de los consumidores cambiaron significativamente en los dos test, demostrando que hay una influencia del conocimiento de la marca sobre la respuesta del consumidor. Cuando se presentan las muestras sin información los consumidores no son influenciados y evalúan el producto en relación a sus características sensoriales. Cuando el nombre de la marca de pan tajado blanco es conocido genera una 
influencia en la aceptación sensorial del producto, es decir, el consumidor aumenta o disminuye su valoración en relación al conocimiento o posicionamiento de la marca en el mercado.

Los consumidores tienen expectativas a determinadas marcas comerciales, de tal forma que la información en ellas genera diferentes efectos. En el caso donde el mercadeo de una marca que tiene una reputación de ofrecer productos de la calidad la asimilación será positivo, en el otro caso la respuesta de los consumidores será negativa.

\section{REFERENCIAS}

Aaker, D., Gestión del valor de la marca. Capitalizar el valor de la marca, Díaz de Santos, Madrid (1994).

Cotes, A. y Muñoz, P. Preferencias de los consumidores de marcas de distribuidor en el mercado del jamón ibérico. Rev. Distribución y Consumo (en línea), http://www.mercasa.es/files/multimedios/4.pdf 53: 52-60 (2010). Acceso 25 de agosto 2013.

De Souza, M., Gonçalves, M., Rezende, C., Dos Santos, C., Rodrigues, V. y Barros, C. Farinhas de soja sem lipoxigenase agregam valor sensorial em bolos. Rev. Ceres, Viçosa, 57(2): 141-144 (2010).

Drake, S., Lopetcharat, K. y Drake, M. Comparison of two methods to explore consumer preferences for cottage cheese. Journal of Dairy Science 92(12):5883-5897 (2009).

Foxall, G.R., Oliveira-Castro, J., James, V.K. y Schrezenmaier, T.C. The behavioral economics of brand choice. London and New York: Palgrave Macmillan (2007).

Grace, P., Ling, L. y Mohd, J. The influence of consumer characteristics on the acceptance of new seaweed food products. Jurnal Kemanusiaan 15: 98-107 (2010).

Hui, Y., Handbook of food science, technology, and engineering. En H. S. Flanagan, Sensory Science: Measuring Consumer Acceptance, CRC Press. (26): 1-23 (2006).

López, D., Sandoval, M. y Cortés, O. Relation between the TRP'S levels, the measure of Brand awareness and buying behavior in Colombian consumers. Psicología desde el Caribe. Universidad del Norte, ISSN 0123-417X (en línea), http://rcientificas.uninorte.edu.co/index.php/psicologia/article/view/45/674, 25: 30-55 (2010). Acceso 25 de agosto 2013.

MacKay, D., Probabilistic scaling analyses of sensory profile, instrumental and hedonic data. J. Chemometrics 19: 180-190 (2005).

Minim, V. Análise Sensorial - Estudo com Consumidores. 2 ed. Viçosa, M.G. Editora da Universidade Federal de Viçosa, (2010).

Minim, V., Milagres, M., Silva, R., Vasconcelos, C., Martins, E. y Sampaio, S. Análise de Risco na Avaliação da Influência da Marca na Aceitabilidade não Sensorial de Requeijão Cremoso. Rev. Inst. Latic. Cândido Tostes 387(67): 79-85 (2012).

Monaco, R., Cavella, S., Di Marzo, S. y Masi, P. The effect of expectations generated by brand name on the acceptability of dried semolina pasta. Food Quality and Preference 15: 429-437 (2004).

Nestrud, M. y Lawless, H. Perceptual mapping of citrus juices using projective mapping and profiling data from culinary professionals and consumers. Food Qual. Prefer. 19:431-438 (2008).

NTC 3884, Instituto Colombiano de Normas Técnicas y Certificación, Análisis Sensorial: Guía General para el Diseño de Cuartos de Prueba, 1-16, Bogotá-Colombia (1996).

Pages, J., Collection and analysis of perceived product interdistances using multiple factor analysis: Application to the study of 10 white wines from the Loire Valley. Food Qual. Prefer. 16:642-649 (2005).

Reicks, A. Consumer Motivations and the Impact of Brand on Purchasing Preferences of Fresh Beef, Doctor of Philosophy, Texas Tech University, Texas (2006).

Semeijn, J., Van Riel, A. y Ambrosin, B. Consumer evaluations of store brands: effects of store image and product attributes. Journal of Retailing and Consumer Services 11: 247-258 (2004).

Sigurdsson, V., Saevarsson, H. y Foxall, G. Brand placement and consumer choice: an in-store experiment. Journal of Applied Behavior Analysis 42(3): 741-745 (2009).

Viana, E., Lopes, J., Cardoso, R., Damasceno, M. y Kersul, C., 2012. Caracterização fisico-química e sensorial de geléia de mamão com araçá-boi. Rev. Bras. Frutic., Jaboticabal - SP 34(4): 1154-1164 (2012). 